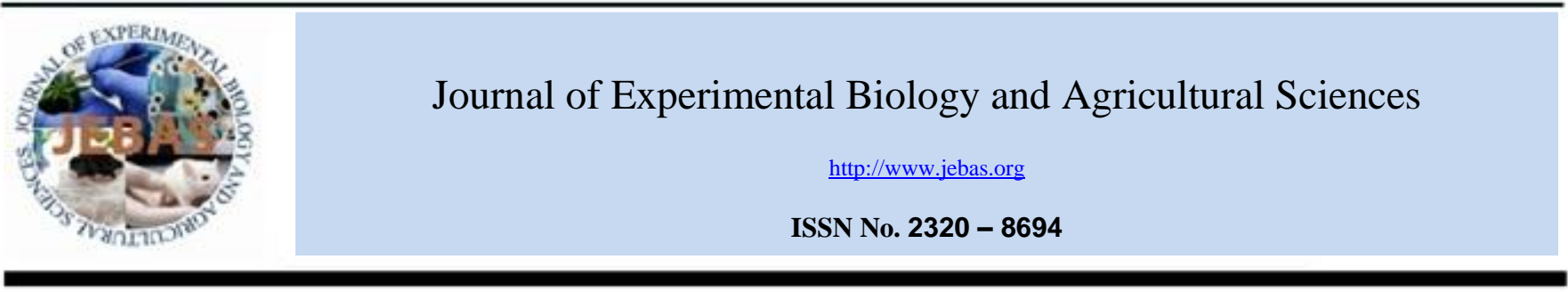

\title{
COMPARATIVE STUDY OF TWO RICE CULTIVARS (Oryza glaberrima and $O$. sativa) UNDER DIFFERENT CULTURAL CONDITIONS
}

\author{
Montcho $\mathrm{D}^{1, *}$, Gbenou $\mathrm{P}^{1}$, Missihoun $\mathrm{AA}^{2}$, Futakuchi $\mathrm{K}^{3}$, Ahanhanzo $\mathrm{C}^{2}$ and Agbangla $\mathrm{C}^{2}$ \\ ${ }^{1}$ National University of Agriculture, BP 43 Ketou, Benin \\ ${ }^{2}$ University of Abomey-Calavi, BP 526 Abomey-Calavi, Benin \\ ${ }^{3}$ Africa Rice Center (AfricaRice), 01 BP 2031 Cotonou, Benin
}

Received - September 06, 2016; Revision - November 13, 2016; Accepted - January 18, 2017

Available Online - February 28, 2017

DOI: http://dx.doi.org/10.18006/2017.5(1).045.053

\begin{abstract}
KEYWORDS
O. glaberrima

O. sativa

Core collection

Toposequence

Variability

ABSTRACT

This study was conducted to evaluate and compare the growth performance of two rice species viz Oryza glaberrima and $O$. sativa under different hydrological conditions. For this, 21 accessions of $O$. glaberrima and 69 of $O$. sativa core collection were cultivated in different water regimes ranging from drought-prone upland to lowland using a toposequence (rainfed upland, rainfed hydromorphic and irrigated lowland) at the experimental field of Africa Rice, Ouedeme, Bénin, during 2010 and 2011 wet seasons. Results of study revealed higher Morpho-physiological and phenological variability among the core collections of $O$. sativa and $O$. glaberrima genotypes. Further, among various studied attributes, $O$. glaberrima showed higher number of tillers at maturity as compared to O. sativa in the second season. Moreover, O. glaberrima also showed long crop duration as compared to $O$. sativa. Leaf area and specific leaf area discriminated $O$. sativa from $O$. glaberrima in all ecologies. Further, $O$. glaberrima also indicated higher leaf area on every sampling date. In case of plant height, non-significant difference was not reported for both species. Variation in results between the two seasons could be justified by the difference between average rainfalls.
\end{abstract}

* Corresponding author

E-mail: montchodav@yahoo.fr (David Montcho)

Peer review under responsibility of Journal of Experimental Biology and Agricultural Sciences.

Production and Hosting by Horizon Publisher India [HPI] (http://www.horizonpublisherindia.in/).

All rights reserved.
All the article published by Journal of Experimental Biology and Agricultural Sciences is licensed under a Creative Commons Attribution-NonCommercial 4.0 International License Based on a work at www.jebas.org.

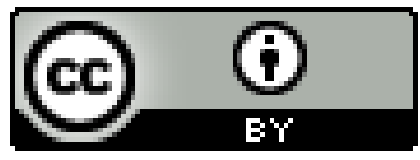




\section{Introduction}

Rice (Oryza sp.) is a major stable food crop in West Africa. Recently, It became a strategic commodity in Africa but its availability and prices are the two major determinants for the welfare of poor consumers threatened by food insecurity (Nwanze et al., 2006; Saito et al., 2010). It grows and widely consumed by more than 40 African countries where about 20 million farmers are engaged in its production and about 100 million peoples directly or indirectly depend on the rice production (Nwanze et al., 2006). Genus Oryza comprises 22 species but among these only two i.e. O. sativa or Asian Rice and $O$. glaberrima or African Rice were cultivated throughout the world. Among this, O. sativa was domesticated more than 10,000 years ago (Kovach et al., 2007) and now it is widely cultivated in various ecologies of the world.

According to Harlan \& Stemler (1976) this variety introduced in Africa about 2,000 years ago. This specie contains different subspecies, among these $O$. sativa var japonicais is well adopted for upland ecosystem while $O$. sativa var indica performing well under various other ecosystems (Kato, 1930). Compared to $O$. sativa, O. glaberrima has short domestication history and according to Linares (2002) this species introduced about 2000-3000 years backs. It can be classified in two ecological groups viz upland and lowland ecosystems (Oka, 1974). Like O. sativa, O. glaberrima does not have any subgroups (Morishima et al.,1962). To argue this statement, Futakuchi \&Sie (2009) concluded that some upland cultivar of O. glaberrima has resistances to lowland constraints. Further, several studies have clarified that these difference between $O$. sativa and $O$. glaberrima may based on various morphological characters (Morishima et al., 1962) such as short ligule, glabrous leaves in $O$. glaberrima and long ligule in the $O$. sativa. Knowledge of comparison between $O$. sativa and $\mathrm{O}$. glaberrima is limited with morpho-physiological and phenological traits. The present study aims to compare $O$. glaberrima and $O$. sativa through different hydrological conditions.

\section{Materials and Methods}

Sixty nine (69) verities of $O$. sativa germplasm was used in this study, these were the core collection of Kojima et al. (2005) and developed at National Institute of Agrobiological sciences, Japan while the twenty-one (21) accessions of $O$. glaberrima were collected from Africa Rice gene bank. Table 1 listed the various accessions which were used in present study. Seed of all genotypes were sown in artificially developed three ecosystems (upland, no flooded hydromorphic and irrigated lowland) at the experimental field of Africa Rice, Ouedeme $\left(6^{\circ} 42^{\prime} 46^{\prime \prime} \mathrm{N}, 1^{\circ} 41^{\prime} 07^{\prime \prime} \mathrm{E}\right)$. The experimental design was Alpha Lattice with three replications of each ecosystem. Nine incomplete blocks were considered in each replicate and ten genotypes in each block. The size of plot was $2 \mathrm{~m}^{2}$ and the space between plants to plants was $25 \mathrm{~cm}$. The experimental plots were fertilized by recommended dose of NPK (15-15-15) which was applied @ $200 \mathrm{~kg} / \mathrm{ha}$ at 25 days after sowing (DAS) and $50 \mathrm{~kg} / \mathrm{ha}$ urea was applied at 40 DAS.

All the necessary agronomic practices recommended for rice have been carried out timely as per standard procedure. In the upland and hydromorphic ecosystems, dry seeds were sown while in lowland cultivation, pre-germinated seeds were sown in a nearly seedbed nursery and transplanted into the main plots at 18 DAS. The vegetative vigor data were recorded in all ecosystems at 30 DAS while plant height and tiller number were recorded at the maturity. Days to maturity was visually assessed on a daily basis starting from booting stage. Data collections have been done according to SES developed by IRRI (1996). Leaf area (LA) was measured by using leaf area meter (LI-3000, LI-COR) at 31DAS, 46DAS and 56DAS. Specific leaf area (SLA) at each sampling date was calculated dividing LA by leaf dry weight. Statistical data analysis was performed using GENSTAT (2003) to compare $O$. sativa and O. glaberrima for all variables involved in this study.

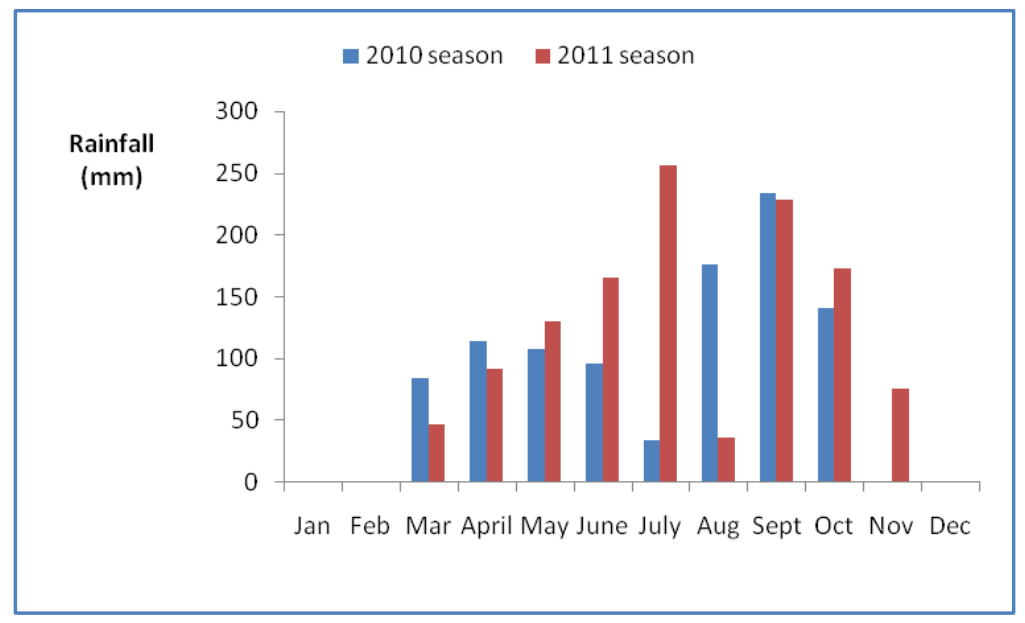

Figure 1 Rainfall distribution in 2010 and 2011. 
Table 1 List of the $O$. sativa and $O$. glaberrima cultivars used in present study

\begin{tabular}{|c|c|c|c|c|c|c|c|}
\hline S. No. & GENOTYPES & $\mathrm{WRC} \mathrm{N}^{0}$ & ORIGINS & S. No. & GENOTYPES & WRC $\mathbf{N}^{0}$ & ORIGINS \\
\hline \multicolumn{8}{|c|}{ O. sativa (core collection) } \\
\hline 1. & NIPPONBARE & WRC 01 & JAPAN & 36. & SURJAMUKHI & WRC 33 & INDIA \\
\hline 2. & KASALATH & WRC 02 & INDIA & 37. & ARC 7291 & WRC 34 & INDIA \\
\hline 3. & BEI KHE & WRC 03 & CAMBODIA & 38. & ARC 5955 & WRC 35 & INDIA \\
\hline 4. & JENA 035 & WRC 04 & NEPAL & 39. & RATUL & WRC 36 & INDIA \\
\hline 5. & NABA & WRC 05 & INDIA & 40. & ARC 7047 & WRC 37 & INDIA \\
\hline 6. & PULUIK ARANG & WRC 06 & INDONESIA & 41. & ARC 11094 & WRC 38 & INDIA \\
\hline 7. & DAVAO 1 & WRC 07 & PHILIPPINES & 42. & BADARI DHAN & WRC 39 & NEPAL \\
\hline 8. & $\begin{array}{ll}\text { RYOU } & \text { SUISAN } \\
\text { KOUMAI } & \\
\end{array}$ & WRC 09 & CHINA & 43. & NEPAL 555 & WRC 40 & INDIA \\
\hline 9. & SHUUSOUSHU & WRC 10 & CHINA & 44. & KALUHEENATI & WRC 41 & SRILANKA \\
\hline 10. & JINGUOYIN & WRC 11 & CHINA & 45. & LOCAL BASMATI & WRC 42 & INDIA \\
\hline 11. & DAHONGGU & WRC 12 & CHINA & 46. & DIANYU 1 & WRC 43 & CHINA \\
\hline 12. & $\mathrm{ASU}$ & WRC 13 & BHUTAN & 47. & BASILANON & WRC 44 & PHILIPPINES \\
\hline 13. & IR 58 & WRC 14 & PHILIPPINES & 48. & MA SHO & WRC 45 & MYANMAR(BURMA) \\
\hline 14. & $\mathrm{CO} 13$ & WRC 15 & INDIA & 49. & KHAO NOK & WRC 46 & LAOS \\
\hline 15. & VARY FUTSI & WRC 16 & MADAGASCAR & 50 & JAGUARY & WRC 47 & BRAZIL \\
\hline 16. & KEIBOBA & WRC 17 & CHINA & 51. & KHAU MAC KHO & WRC 48 & VIETNAM \\
\hline 17. & QINGYU(SEIYU) & WRC 18 & TAIWAN & 52. & PADI PERAK & WRC 49 & INDONESIA \\
\hline 18. & DENG PAO ZHAI & WRC 19 & CHINA & 53. & REXMONT & WRC 50 & USA \\
\hline 19. & TADUKAN & WRC 20 & PHILIPPINES & 54. & URASAN 1 & WRC 51 & JAPAN \\
\hline 20. & SHWE NANG GYI & WRC 21 & MYANMAR(BURMA) & 55. & KHAU TAN CHIEM & WRC 52 & VIETNAM \\
\hline 21. & CALOTOC & WRC 22 & PHILIPPINES & 56. & TIMA & WRC 53 & BHUTAN \\
\hline 22. & LEBED & WRC 23 & PHILIPPINES & 57. & TUPA 729 & WRC 55 & BANGLADESH \\
\hline 23. & PINULUPOT 1 & WRC 24 & PHILIPPINES & 58. & MILYANG 23 & WRC 57 & REP.KOREA \\
\hline 24. & MUHA & WRC 25 & INDIA & 59. & NEANG MENH & WRC 58 & CAMBODIA \\
\hline 25. & JHONA 2 & WRC 26 & INDIA & 60. & NEANG PHTONG & WRC 59 & CAMBODIA \\
\hline 26. & NEPAL 8 & WRC 27 & NEPAL & 61. & HAKPHAYNHAY & WRC 60 & LAOS \\
\hline 27. & JARJAN & WRC 28 & BHUTAN & 62. & RADIN GOI SESAT & WRC 61 & MALAYSIA \\
\hline 28. & KALO DHAN & WRC 29 & NEPAL & 63. & KEMASIN & WRC 62 & MALAYSIA \\
\hline 29. & ANJANA DHAN & WRC 30 & NEPAL & 64. & BLEIYO & WRC 63 & THAILAND \\
\hline 30. & SHONI & WRC 31 & BANGLADESH & 65. & PADI KUNING & WRC 64 & INDONESIA \\
\hline 31. & TUPA 121-3 & WRC 32 & BANGLADESH & 66. & RAMBHOG & WRC 65 & INDONESIA \\
\hline 32. & BINGALA & WRC 66 & MYANMAR(BURMA) & 67. & CHIN GALAY & WRC 97 & MYANMAR(BURMA) \\
\hline 33. & PHULBA & WRC 67 & INDIA & 68. & DEEJIAOHUALUO & WRC 98 & CHINA \\
\hline 34. & KHAO NAM JEN & WRC 68 & LAOS & 69. & HONG CHEUH ZAI & WRC 99 & CHINA \\
\hline 35. & VANDARAN & WRC 100 & SRILANKA & - & - & - & - \\
\hline \multirow[t]{2}{*}{ S. No. } & GENOTYPES & WRC No $^{0}$ & ORIGINS & S. No. & GENOTYPES & WRC N $^{0}$ & ORIGINS \\
\hline & O. glaberrima & & & & & & \\
\hline 1. & CG 14 & - & SENEGAL & 12. & TOG 6748 & - & LIBERIA \\
\hline 2. & CG 17 & - & SENEGAL & 13. & TOG 6804 & - & NIGERIA \\
\hline 3. & CG 20 & - & SENEGAL & 14. & TOG 7206 & - & COTE D'IVOIRE \\
\hline 4. & TOG 12303 & - & COTE D'IVOIRE & 15. & TOG 7420 & - & SIERRA LEONE \\
\hline 5. & TOG 5473 & - & NIGERIA & 16. & TOG 7442 & - & NIGERIA \\
\hline 6. & TOG 5641 & - & NIGERIA & 17. & TOG 9066 & - & NIGERIA \\
\hline 7. & TOG 5681 & - & NIGERIA & 18. & TOG 9276 & - & NIGERIA \\
\hline 8. & TOG 5775 & - & LIBERIA & 19. & TOG 9280 & - & NIGERIA \\
\hline 9. & TOG 5829 & - & LIBERIA & 20. & TOG 9281 & - & NIGERIA \\
\hline 10. & TOG 6740 & - & LIBERIA & 21. & TOG 9300 & - & NIGERIA \\
\hline 11. & SALIFOREH & - & SIERRA LEONE & & & & \\
\hline
\end{tabular}


Table 2 Phenotypic frequency of vegetative vigor for both species in all ecosystems (\%).

\begin{tabular}{|c|c|c|c|c|c|c|}
\hline \multirow{2}{*}{$\begin{array}{l}\text { Ecologies } \\
\text { Classes } \\
\end{array}$} & \multicolumn{2}{|l|}{ Upland } & \multicolumn{2}{|c|}{ Hydromorphic } & \multicolumn{2}{|l|}{ Lowland } \\
\hline & O. sativa & O. glaberrima & O. sativa & O. glaberrima & O. sativa & O. glaberrima \\
\hline \multicolumn{7}{|l|}{2010 season } \\
\hline Extra vigorous & 42.03 & 57.14 & 68.12 & 80.95 & 0 & 0 \\
\hline Vigorous & 53.62 & 38.10 & 28.98 & 19.05 & 20.29 & 23.81 \\
\hline normal & 4.35 & 4.76 & 2.90 & 0 & 71.01 & 71.43 \\
\hline weak & 0 & 0 & 0 & 0 & 8.70 & 4.76 \\
\hline Very weak & 0 & 0 & 0 & 0 & 0 & 0 \\
\hline \multicolumn{7}{|l|}{2011 season } \\
\hline Extra vigorous & 43.48 & 61.90 & 43.48 & 71.43 & 5.80 & 9.52 \\
\hline Vigorous & 47.83 & 38.10 & 43.48 & 28.57 & 39.13 & 38.10 \\
\hline Normal & 8.70 & 0 & 13.04 & 0 & 55.07 & 52.38 \\
\hline Weak & 0 & 0 & 0 & 0 & 0 & 0 \\
\hline Very weak & 0 & 0 & 0 & 0 & 0 & 0 \\
\hline
\end{tabular}

\section{Results}

Results of study revealed a typical bimodal Rainfall distribution pattern for the experimental site. During cropping season, lowest rainfalls were reported in July, 2010 cropping season while it was reported lowest in August of 2011cropping season. Figure 1 showed rainfall distribution during crop seasons of 2010 and 2011.

Comparison between $O$. glaberrima and $O$. sativa was made for each variable in each ecosystem for both years. Vegetative vigor was scored between 1 (extra vigorous) to 5 (normal vigor) for $O$. sativa in all ecological conditions of both years for both cropping seasons. Therefore plant grown in upland ecological conditions shows higher vigorous characteristics (score 3 ) and shows 53.62 and 47.83 percent vigorous effect in 2010 and 2011 respectively. Highest vigorous percentages 68.12 and 43.48 were reported under hydromorphic ecological conditions of cropping season of 2010 and 2011 respectively. Under lowland ecosystem condition normal vigor showed
$71.01 \%$ vigorous characteristics in 2010 while this percentage was reported $55.07 \%$ for the cropping season of 2011 .

Vegetative vigor of $O$. glaberrima was reported between 1 (extra vigorous) to 3 (vigorous) for upland and hydromorphic ecologies. For $O$. glaberrima, vigorous nature was reported $95.24 \%$ for upland ecosystem conditions while this value reached $100 \%$ for hydromorphic agro-ecosystem and these two are not significantly different. This vigorous nature reached $100 \%$ for both the ecosystem in the second season. In lowland ecosystem, vegetative vigor was reported between 3 (vigorous) to 7 (weak) for $O$. glaberrima germplasm and the normal vigor had the high percentage $71.43 \%$ in 2010 . While in 2011, it was scored between 1 (extra vigorous) to 5 (normal) and the normal vigor percentage was reported $52.38 \%$ (table 2). No significant difference was found between $O$. sativa and $O$. glaberrima for plant height at maturity in all studied agricultural agroecosystem both seasons. In lowland ecology ( $\mathrm{P}=0.544), O$. glaberrima showed $149.9 \mathrm{~cm}$ plant height and this value was reported $147.6 \mathrm{~cm}$ for $O$. sativa.

Table 3 Phenotypic Frequency of plant height for both species in all ecosystems (\%).

\begin{tabular}{|c|c|c|c|c|c|c|}
\hline \multirow{2}{*}{$\begin{array}{l}\text { Ecologies } \\
\text { Classes }\end{array}$} & \multicolumn{2}{|l|}{ Upland } & \multicolumn{2}{|c|}{ Hydromorphic } & \multicolumn{2}{|l|}{ Lowland } \\
\hline & O.sativa & O. glaberrima & O. sativa & O. glaberrima & O. sativa & O. glaberrima \\
\hline \multicolumn{7}{|l|}{2010 season } \\
\hline Semi dwarf & 20.29 & 0 & 13.04 & 0 & 10.14 & 0 \\
\hline Intermediate & 26.09 & 42.86 & 17.39 & 14.29 & 11.59 & 4.76 \\
\hline Tall & 53.62 & 57.14 & 69.57 & 85.71 & 78.26 & 95.24 \\
\hline \multicolumn{7}{|l|}{2011 season } \\
\hline Semi dwarf & 36.23 & 28.57 & 26.09 & 14.29 & 7.25 & 0 \\
\hline Intermediate & 44.93 & 61.90 & 50.72 & 57.14 & 4.35 & 0 \\
\hline Tall & 18.84 & 9.52 & 23.19 & 28.57 & 88.41 & 100 \\
\hline
\end{tabular}



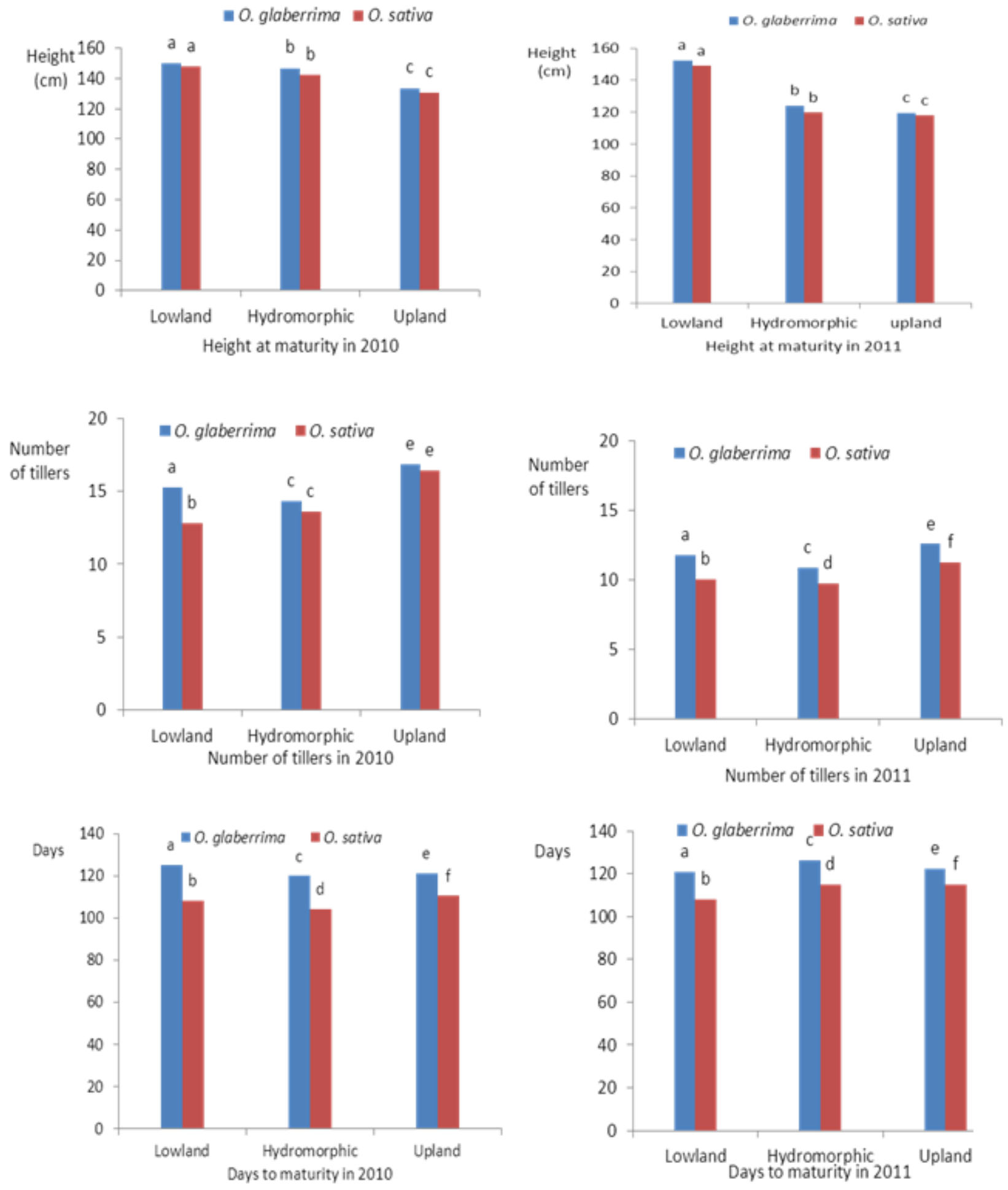

Figure 2 Comparison of $O$. glaberrima and $O$. sativa in all ecosystems 
Table 4 Phenotypic frequency for tillers number for both species in all ecosystem (\%).

\begin{tabular}{|c|c|c|c|c|c|c|}
\hline \multirow{2}{*}{$\begin{array}{l}\text { Ecologies } \\
\text { Classes }\end{array}$} & \multicolumn{2}{|l|}{ Upland } & \multicolumn{2}{|c|}{ Hydromorphic } & \multicolumn{2}{|l|}{ Lowland } \\
\hline & $O$ sativa & O. glaberrima & $O$ sativa & O. glaberrima & $O$ sativa & O. glaberrima \\
\hline \multicolumn{7}{|c|}{2010 season } \\
\hline Very high & 2.90 & 0 & 1.45 & 0 & 0 & 0 \\
\hline Good & 18.84 & 14.29 & 5.80 & 4.76 & 2.90 & 4.76 \\
\hline Medium & 76.81 & 85.71 & 75.36 & 90.48 & 79.71 & 95.24 \\
\hline Low & 1.45 & 0 & 17.39 & 4.76 & 17.39 & 0 \\
\hline Very low & 0 & 0 & 0 & 0 & 0 & 0 \\
\hline \multicolumn{7}{|c|}{2011 season } \\
\hline Very high & 1.45 & 0 & 0 & 0 & 0 & 0 \\
\hline High & 1.45 & 4.76 & 0 & 0 & 1.45 & 0 \\
\hline Medium & 66.67 & 80.95 & 47.83 & 80.95 & 49.28 & 80.95 \\
\hline Low & 30.43 & 14.29 & 52.17 & 19.05 & 47.82 & 19.05 \\
\hline Very low & 0 & 0 & 0 & 0 & 1.45 & 0 \\
\hline
\end{tabular}

In hydromorphic ecosystem $(\mathrm{P}=0.235)$ this plant height was reported $146.67 \mathrm{~cm}$ for $O$. glaberrima and $142.24 \mathrm{~cm}$ for $O$. sativa while in upland ecology $(\mathrm{P}=0.468), O$. glaberrima showed $133.26 \mathrm{~cm}$ and $O$. sativa gave $130.7 \mathrm{~cm}$ hight. Further, in the second season, similar result was found for plant height and $O$. glaberrima and $O$. sativa (Figure 2).

Scores of plant height for $O$. sativa were comprised between 1 (semi dwarf) to 9 (tall) in both seasons in all ecologies. In first season, $53.62 \%$ genotypes of the $O$. sativa were tall in upland, while this value was reported between $69.57 \%$ for hydromorphic and $78.26 \%$ for lowland ecosystems. The second season showed majority of plants as intermediate in upland $(44.93 \%)$ and hydromorphic $(50.72 \%)$ while for In lowland ecosystem, $88.41 \%$ of the $O$. sativa plants were tall. Similar pattern was reported for $O$. glaberrima and $57.14 \%$ genotypes were tall for upland, while it was reported $85.71 \%$ for hydromorphic and $95.24 \%$ for lowland. In the second season of $2011,61.90 \%$ O. glaberrima genotypes grown in the upland are tall, while this percentage was 57.14 and $100 \%$ for plant grown in hydromorphic and lowland ecosystem (Table $3)$.
In case of tillers number at maturity, a significant difference $(\mathrm{P}<0.001)$ was reported between $O$. glaberrima $(15.32$ tillers/plant) and $O$. sativa (12.80 tillers/plant) at lowland ecosystem in the first season of 2010. While, in case of upland $(\mathrm{P}=0.250)$ and hydromorphic $(\mathrm{P}=0.489)$, no significant difference was found between $O$. glaberrima and $O$. sativa. While, in the second season significant difference $(\mathrm{P}<0.05)$ was observed between $O$. glaberrima and $O$. sativa in all ecologies. Means values of $O$. glaberrima were higher as compared to $O$. sativa. Concerning the number of tillers, $O$. glaberrima have 11.78 tillers/plant in lowland while this number was 10.88 and 12.59 tillers/plant for hydromorphic and upland ecosystem respectively. In case of $O$. sativa genotypes, tiller number was 10.04, 9.71 and 11.21 tillers/plant for lowland, hydromorphic and upland respectively (Figure 1). The scores of tiller number in the first season were ranged from 1(very high) to 7 (low) for $O$. sativa while, in case of $O$. glaberrima tiller number showed two classes viz 3 (good) and 5 (medium). Percentage tiller was reported 76.81, 75.36 and $79.71 \%$ for upland, hydromorphic and lowland ecosystem. Similar results were obtained in the second season of 2011 (Table 4).

Table 5 Phenotypic Frequency of days to maturity for both species in all ecosystem (\%).

\begin{tabular}{|lllllll|}
\hline Ecologies & Upland & \multicolumn{2}{l}{ Hydromorphic } & Lowland \\
\hline Classes & O. sativa & O. glaberrima & O. sativa & O. glaberrima & O. sativa & O. glaberrima \\
\hline 2010 season & & & & & & 4.76 \\
\hline Very early & 24.64 & 9.52 & 56.52 & 19.05 & 39.13 & 23.81 \\
\hline Early & 56.52 & 33.33 & 28.99 & 14.29 & 44.93 & 28.57 \\
\hline Medium & 8.70 & 28.57 & 5.80 & 38.10 & 7.25 & 42.86 \\
\hline Late & 2.90 & 28.57 & 2.90 & 28.57 & 4.35 & 0 \\
\hline Very late & 7.25 & 0 & 5.80 & 0 & 4.35 & 4.76 \\
\hline 2011 season & & & & 28.99 & 23.81 \\
\hline Very early & 27.54 & 4.76 & 17.39 & 9.52 & 49.28 & 57.14 \\
\hline Early & 36.23 & 14.29 & 44.93 & 14.29 & 13.04 & 14.29 \\
\hline Medium & 20.29 & 52.38 & 23.19 & 33.33 & 4.35 \\
\hline Late & 7.25 & 28.57 & 2.90 & 38.10 & 4.35 \\
\hline Very late & 8.70 & 0 & 11.59 & 4.76 & \\
\hline
\end{tabular}


Table 6 Time courses of leaf area for both species in all ecologies $\left(\mathrm{cm}^{2}\right)$.

\begin{tabular}{|lccccccccc|} 
& \multicolumn{3}{c}{ Upland } & \multicolumn{3}{c}{ Hydromorphic } & \multicolumn{2}{c|}{ Lowland } \\
\hline & 31DAS & 46DAS & 56DAS & 31DAS & 46DAS & 56DAS & 31DAS & 46DAS & 56DAS \\
\hline 2010 season & & & & & & & & & \\
\hline O. glaberrima & 273.8 & 749.51 & 1182.7 & 335.27 & 629.2 & 1161 & 121.02 & 485.7 & 1108.4 \\
\hline O. sativa & 229.8 & 557.55 & 935.65 & 251.04 & 490.5 & 906 & 79.33 & 305.4 & 875.4 \\
\hline Probability & 0.018 & $<0.001$ & $<0.001$ & $<0.001$ & $<0.001$ & $<0.001$ & $<0.001$ & $<0.001$ & $<0.001$ \\
\hline 2011 season & & & & & & & & & \\
\hline O. glaberrima & 173.35 & 586.9 & 1022.3 & 94.01 & 492.8 & 715.9 & 41.67 & 397.98 & 1263.84 \\
\hline O. sativa & 111.36 & 401.1 & 717.5 & 66.12 & 295.7 & 451.4 & 36.62 & 302.05 & 933.87 \\
\hline Probability & $<0.001$ & $<0.001$ & $<0.001$ & $<0.001$ & $<0.001$ & $<0.001$ & 0.071 & $<0.001$ & $<0.001$ \\
\hline
\end{tabular}

Like other parameters, maturity also showed highly significant difference $(\mathrm{P}<0.001)$ between $O$. glaberrima and $O$. sativa for all ecologies in both seasons. In the first season, in lowland means values indicated 125.4 days for O. glaberrima and 108.4 days for $O$. sativa. Further, for $O$. glaberrima maturity time was reported 120 days under hydromorphic ecosystem and this duration was reported 104.4 days for $O$. sativa in under same ecosystem. Under upland ecosystem conditions, means maturity time was reported 121.3 days for $O$. glaberrima while this was reported 110.6 days for $O$. sativa. In 2011, no significant differences was reported amongst the various ecosystem and means maturity time under lowland condition was reported 121 days for $O$. glaberrima and 107.9 days for $O$. sativa while this maturing time was 126.4 days for $O$. glaberrima and 114.8 days for $O$. sativa under hydromorphic ecosystem. In upland ecosystem conditions, maturity time was reported 122.4 and 115 days for $O$. glaberrima and $O$. sativa respectively (Figure 1). Days to maturity was scored between 1 (very early) to 9 (very late) for $O$. sativa and between 1 (very early) to 7 (late) for $O$. glaberrima in all ecologies in both seasons. In the first season high percentage $(56.52 \%)$ had early maturing class in upland and in lowland (44.93\%) for O. sativa but for O. glaberrima high percentage $(56.52 \%)$ was revealed by very early genotypes under hydromorphic ecosystem while high percentage in early maturing class $33.33 \%$ in upland ecology. In 2011 high percentage was observed in early maturing class at all ecologies and it was reported $36.23 \%$ in upland, $44.93 \%$ in hydromorphic and $49.28 \%$ in lowland for $O$. sativa. While, intermediate early maturity was reported for $O$. glaberrima in upland $(52.38 \%)$ and lowland $(57.14 \%)$ ecosystem but $O$. glaberrima genotypes grown in hydromrphic ecosystem shows high percentage $(38.10 \%)$ of late maturing ( Table 5).

Table 6 shows time courses of leaf area for all ecologies tested in both seasons. An expansion in Leaf area was reported from 31 to 56 DAS for both $O$. glaberrima and $O$. sativa. The comparison of both species revealed that leaf area of $O$. glaberrima was higher than $O$. sativa on each sampling date. Same pattern was obtained in all ecologies in both seasons. Means of specific leaf area (SLA) decreased gradually between 31 and 56 DAS and uniform among ecologies for $O$. glaberrima and $O$. sativa. O. glaberrima had the greatest SLA whereas $O$. sativa had the smallest at each sampling date (Table 7).

\section{Discussion}

This is the first attempt to compare core collection of $O$. sativa to $O$. glaberrima under different hydrological conditions by using morpho-physiological and phenological variables. Present study has been taken for the whole diversity of $O$. sativa developed by Kojima et al (2005) in NIAS Institute in Japan. As per the Frankel (1984) suggestion, development of core collection is important for the effective uses of germplasms. High variability ( 1 to 7 ) was observed within the $O$. glaberrima and $O$. sativa vegetative vigor in all ecosystems. Plant height at maturity did not discriminate $O$. glaberrima and $O$. sativa as shown by non-significant probability. Semi dwarf, intermediate and tall genotypes were reported in both species. In the each ecosystem, plant height is not discriminate variable.

Table 7 Time courses of specific leaf area for both species in all ecologies $\mathrm{m}^{2} \mathrm{~kg}^{-1}$.

\begin{tabular}{|llllllllll|}
\hline & Upland & \multicolumn{9}{c|}{ hydromorphic } & \multicolumn{3}{c|}{ lowland } \\
\hline & 31DAS & 46DAS & 56DAS & 31DAS & 46DAS & 56DAS & 31DAS & 46DAS & 56DAS \\
\hline 2010 season & & & & & & & & & \\
\hline O. glaberrima & 37.22 & 30.68 & 21.86 & 36.50 & 29.95 & 22.70 & 37.6 & 30.38 & 22.53 \\
\hline O. sstiva & 27.54 & 22.76 & 17.09 & 26.96 & 21.98 & 17.07 & 26.97 & 21.37 & 18.67 \\
\hline Probability & $<0.001$ & $<0.001$ & $<0.001$ & $<0.001$ & $<0.001$ & $<0.001$ & 0.002 & $<0.001$ & $<0.001$ \\
\hline 2011 season & & & & & & & & & 21 \\
\hline O. glaberrima & 37.25 & 30.22 & 24.13 & 36.31 & 28.76 & 22.39 & 35.79 & 29.33 & 21.53 \\
\hline O. sativa & 30.01 & 25.22 & 20.22 & 32.75 & 25.15 & 20.86 & 32.07 & 26.15 & 18.67 \\
\hline Probability & $<0.001$ & $<0.001$ & $<0.001$ & 0.009 & $<0.001$ & 0.019 & 0.002 & $<0.001$ & $<0.001$ \\
\hline
\end{tabular}


On the other hand number of tillers at maturity, maturity time, leaf area and specific leaf area discriminate well for $O$. glaberrima and $O$. sativa. High tillers number was observed in all geographical area for O. glaberrima in 2011 . Similarly, Dingkuhn et al.(1998) reported about twice tillage production in O. glaberrima (CG 14) as compared to O. sativa (WAB 56104) under upland condition. High tillering capacity is considered as desirable trait in rice production since number of tillers per plant is closely related to number of panicle and grain yield (Wang et al., 2007). Comparison of means days of maturity between $O$. glaberrima and $O$. sativa revealed highly significant difference in all ecosystems.

Result of study revealed that $O$. sativa had shorter duration as compared to $O$. glaberrima. High variability was reported in days to maturity for both species from very early to very late maturing. Crop duration is interactively determined genotype and environment. Crop duration of rice genotype varied strongly in their sensitivity to photoperiod. It depends mainly on the cultivar-specific duration of the basic vegetative phase (Dingkuhn \&Asch, 1999).

Comparison between rice cultivars of different ecological adaptation which were planted across hydrological sequence indicated strong genotype specific effects of the ecosystem on growth and crop duration (Dingkuhn \&Asch, 1999). This study did not go depth to make difference between $O$. sativa japonica and $O$. sativa indica because core collection comprises both subspecies. Therefore it has been well established that $O$. sativa indica has long crop duration and high tillering ability. Similarly, Dingkuhn \&Asch (1999) reported long crop duration under all studied ecologies (upland, hydromorphic and lowland) for O. sativa indica cultivar (Bouake 189) and it was longer than the $O$. glaberrima (CG 14). While the other genotype of $O$. sativa japonica cultivar (WAB 56-105) has shorter crop duration as compared to CG 14. This thing is suggesting that results can vary with the used genotypes.

Special leaf area gradually (SLA) increased between 31 to 56 DAS for $O$. glaberrima and $O$. sativa. Moreover $O$. glaberrima achieve superior SLA as compared to $O$. sativa. SLA has previously been found higher for $O$. glaberrima than $O$. sativa (Dingkuhn et al., 1998; Asch et al., 1999; Dingkuhn et al., 1999; Moukoumbi et al., 2011). SLA despite being a parameter that is strongly affected by sampling date discriminate well $O$. glaberrima and $O$. sativa. A high SLA reduces the amount of assimilates needed to produce a given leaf area, resulting in early ground cover, and therefore a greater light harvest and higher canopy photosynthesis rates early in the season (Dingkuhn et al., 1999). SLA has been shown highly predictive and positive correlation was found with weed competitiveness (Dingkuhn et al., 1999). It is an excellent criterion of breeding for weed competitive. Droopiness of the leaves of many accessions of $O$. glaberrima caused the highest of SLA (Dingkuhn et al., 1998).

\section{Acknowledgment}

This study was financially supported by the Ministry of Foreign Affairs, Japan and the Stress-tolerant Rice for Africa and South Asia Project funded by the Bill and Melinda Gates Foundation and Africa Rice. The authors would like to thank this support. The authors also thank the National Institute of Agrobiological Sciences (NIAS), Japan for providing rice accessions from the NIAS core collection.

\section{Conflict of interest}

Authors would hereby like to declare that there is no conflict of interests that could possibly arise.

\section{References}

Asch F, Sow A, Dingkuhn M (1999) Reserve mobilization, dry matter partitioning and specific leaf area in seedlings of African rice cultivars differing in early vigor. Field Crops Research 62:191-202. DOI: http://dx.doi.org/10.1016/S03784290(99)00020-9.

Dingkuhn M, Asch F (1999) Phenological responses of Oryza sativa, O. glaberrima and inter-specific rice cultivars on a toposquence inWest Africa. Euphytica 110:109 - 126.

Dingkuhn M, Johnson DE, Sow A, Audebert AY (1999) Relationships between upland rice canopy characteristics and weed competitiveness. Field Crops Research 61:79-95. DOI: http://dx.doi.org/10.1016/S0378-4290(98)00152-X.

Dingkuhn M, Jones MP, Johnson DE, Sow A (1998) Growth and yield potential of Oryza sativa and O. glaberrima upland rice cultivars and their interspecific progenies. Field Crops Research 57:57-69. DOI: http://dx.doi.org/10.1016/S03784290(97)00115-9.

Frankel OH (1984) Genetic Perspectives of Germplasm Conservation. In: Arber WK, Llimensee K, Peacock WJ, Stralinger P (Eds.), Genetic Manipulation: Impact on Man and Society, Cambridge University Press, Cambridge, 161-170.

Futakuchi K, Sie M (2009) Better exploitation of African rice (Oryza glaberrima Steud.) in varietal development for resource-poor farmers in West and Central Africa. Agricultural Journal 4:96-102.

GenStat (2003) GenStat for Windows. Release 4.23DE Discovery Edition. VSN International Ltd., Hemel Hempstead, UK.

Harlan JR, Stemler A (1976) The races of Sorghum in Africa. In: Harlan J, de Wet JM, Stemler AB (Eds.), Origin of African plant domestication. Mouton Publishers, The Hague, The Netherlands. 
IRRI (1996) Standard Evaluation System for rice manual.Manila, Philippines 52pp.

Kato S (1930) On the affinity of the cultivated varieties of rice plants, Oryza sativa L. . Journal of the Department of Agriculture, Kyushu Imperial University - Journal of the Faculty of Agriculture, Kyushu University 2 :241 - 276.

Kojima Y, Ebana K, Fukuoka S, Nagamine T, Kawase M (2005) Development of an RFLP-based Rice Diversity Research Set of Germplasm. Breeding Science 55:431 - 440.

Kovach MJ, Sweeney MT, McCouch SR (2007) New insights into the history of rice domestication. Trends in Genetics 23: 578-587. DOI: http://dx.doi.org/10.1016/j.tig.2007.08.012.

Linares OF (2002) African rice (Oryza glaberrima): history and future potential. Proceeding of the National Academy of Science 99:16360-16365. doi: 10.1073/pnas.252604599.

Morishima H, Hinata K, Oka HI (1962) Comparison between two Cultivated Rice Species, Oryza sativa L. and O. glaberrima Steud. Japanese Journal of Breeding 12:152-165. DOI: http://doi.org/10.1270/jsbbs 1951.12.153.
Moukoumbi YD, Sie M, Vodouhe R, Bonou W, Toulou B, Ahanchede A (2011) Screening of rice varieties for their weed competitiveness. African Journal of Agricultural Research 6 : 5446 - 5456. DOI: 10.5897/AJAR11.1162.

Nwanze KF, Mohapatra S, Kormawa P, Keya S, Bruce - Oliver S (2006) Perspective: Rice development in sub-Saharan Africa. Journal of the Science of Food and Agriculture 86:675 - 677. DOI:10.1002/jsfa.2415.

Oka HI (1974) Experimental studies on the origin of cultivated rice. Genetics 78:475-486.

Saito K, Azoma K, Sié M (2010) Grain Yield Performance of Selected Lowland NERICA and Modern Asian Rice Genotypes in West Africa. Crop Science 50:281-291.

Wang F, Cheng F, Zhang GP (2007) Difference in Grain Yield and Quality among Tillers in Rice Genotypes Differing in Tillering Capacity. Rice Science $14: 135-140$. DOI: http://dx.doi.org/10.1016/S1672-6308(07)60019-5. 